\title{
Hybrid Approach for Analyzing SAS Data from Enzyme-Graphene Nanocomposite
}

Volker S. Urban ${ }^{1}$, Durgesh K. Rai ${ }^{2}$, Manickam Gurusaran ${ }^{3}$, Venkatesan Renugopalakrishnan ${ }^{4}$

${ }^{1}$ Biology and Soft Matter Division, Oak Ridge National Laboratory, Oak Ridge, TN, USA, ${ }^{2}$ Nuclear Reactor Laboratory, Massachusetts Institute of Technology, Cambridge, MA, USA, ${ }^{3}$ Department of Computational and Data Sciences, Indian Institute of Science, Bangalore, India, ${ }^{4}$ Center for Life Sciences, Boston Children's Hospital, Harvard Medical School, Boston, MA, USA and Department of Chemistry and Chemical Biology, Northeastern University, Boston, $M A, U S A$.

Many researchers struggle with the challenges of characterizing complex, multi-component systems, such as those found in functional bio-inorganic sensors. Methods are scarce that can discern the conformation of biological molecules while they are imbedded in a biological/synthetic composite material, yet such methods are needed for understanding enzyme functionality in practical applications such as in blood glucose-monitoring biosensors. We report observations of sensor matrix structure obtained from small-angle neutron scattering analysis of graphene-based electrodes functionalized with glucose oxidase (GOx) under different $\mathrm{pH}$ conditions. A methodology to separately extract the average shape of enzyme molecules and their hierarchical assemblies was developed. The modeling is able to resolve differences in the average GOx dimer structure and shows that treatment under different $\mathrm{pH}$ conditions leads to structural differences within the enzyme at the dimer contact region. The coupling of different analysis and modeling approaches based on both scaling arguments and ab-initio shape modeling as developed in this study provides a universal approach to obtain detailed structural quantifications, for establishing robust structure-property relationships. 